\title{
LA MODELACIÓN MATEMÁTICA, UNA ESTRATEGIA PARA LA ENSEÑANZA DE LA ESTADÍSTICA
}

\section{MATHEMATICAL MODELING, A STRATEGY FOR THE EDUCATION OF STATISTICS}

Roberto Carlos Caballero Florez ${ }^{1}$

\section{Marlon de Jesus Rondon Meza²}

\section{Lacides Alfonso Baleta Palomino ${ }^{3}$}

Teovaldo García Romero ${ }^{4}$

Universidad Popular del Cesar

\footnotetext{
$1 \quad$ Candidato a Magister en Ciencias Matemáticas de la Universidad del Atlántico, Licenciado en Matemáticas y Física de la Universidad Popular del Cesar, adscrito como Joven Investigador de la Universidad Popular del Cesar por el proyecto de Investigación Enseñanza de la estadística: un desafío abordado por la modelación matemática. ORCID: https://orcid.org/0000-0002-1649-8179

$2 \quad$ Posdoctorado en didáctica de la investigación científica de inicc-peru, Doctorado en Educación de la Universidad Dr. Rafael Belloso Chacín, Magister en matemáticas de Universidad del Zulia, Licenciado en matemáticas y física de Universidad Popular del Cesar.

ORCID: https://orcid.org/0000-0002-4172-8319

$3 \quad$ Doctorado en Ciencias de la Educación de la Universidad Privada Dr. Rafael Belloso Chacín, Magister en Matemáticas Aplicadas de la Universidad del Zulia, Licenciado En Matemática y Física de la Universidad Popular del Cesar. Investigador Junior ante Colciencias. Universidad Popular del Cesar. https:/orcid.org/0000-0002-3501-2365

4 Doctorado en ciencias de la Universidad Rafael Belloso Chacín, Magister en Gerencia de Proyectos de I+D de Universidad Dr. Rafael Belloso Chacín, Especialista en educación matemática de Universidad de Pamplona, Licenciado en matemáticas y física de la Universidad Popular del Cesar. Investigador Senior ante Colciencias. Adscrito como Docente en la Facultad de Ciencias de la educación de Universidad Popular del Cesar.

ORCID: https://orcid.org/0000-0002-7233-4064
} 


\section{RESUMEN}

Este artículo socializa la experiencia tomada del proyecto "Enseñanza de la estadística: un desafío abordado por la modelación matemática" financiado por la Universidad Popular del Cesar en su convocatoria 2018, aplicada en 2019 con estudiantes de grado 5 de nivel básica primaria, en una institución oficial ubicado en la Jagua de Ibirico zona carbonífera del departamento del Cesar (Colombia).

Se implementa un conjunto de estrategias didácticas centradas en la modelación matemática, con lo cual los estudiantes inician con un esquema de situaciones problémicas de su contexto hasta la construcción de un modelo matemático, centrados en la representación gráfica donde describan cada situación, comparando cada una de ellas y así estas sean familiares o matutinas, para resolver alguna situación real o hipotética que sea común en su cotidianidad. Estos modelos pueden tener problemas que se solucionen interdisciplinariamente y que su relación sea transversal a diferentes áreas del conocimiento. Con el diseño e implementación de estas estrategias para la enseñanza y el aprendizaje de la estadística, el maestro pasa de ser un educador tradicional de las ciencias exactas a tener un rol de docente-investigador y el estudiante asuma un ritmo de aprendizaje según lo propuesto. Los datos obtenidos proporcionaron información que corrobora la hipótesis inicial sobre el rendimiento escolar, puesto que son útiles al proceso de enseñanza de los niños de la básica primaria.

Alrededor de la modelación se vienen trabajando líneas teóricas con el mismo paradigma y diferentes perfiles; el trabajo se apoyó las teorías de Bassanezi (2002) y Biembengut \& Hein (2004), que proponen la construcción de modelos a partir del contexto y del entorno social y cultural del estudiante.
El Ministerio de Educación Nacional (1998, 2006) hace importantes sobre lo significativo que es el proceso de modelación y los involucra en la soluciones de problemas reales que posteriormente pueden ser matematizados $y$ en general a que aquellos aprendizajes sean significativos. En ese sentido Villa (2007) contempla el proceso de modelación acorde con los estándares básicos de competencias, permitiendo fortalecer las pruebas saber, además de la apropiación de los derechos básicos de aprendizaje.

Así mismo en la prueba posterior se encontró un mayor rendimiento de cada estudiante. Por lo tanto se ha logrado generar espacios de comunicación con los docentes de aula, reflexionando sobre las diferentes estrategias utilizadas en el aula para lograr un mejor aprendizaje de los educandos y a su vez sobre el reto en cada uno de ellos en relación al rol investigativo del docente.

PALABRAS CLAVE: modelación matemática, enseñanza, estrategias pedagógicas.

\section{ABSTRACT}

This article socializes the research experience taken from the project "Teaching statistics: a challenge addressed by mathematical modeling" financed by the Popular University of Cesar in its 2018 call, applied in 2019 with students of grade 5 of primary basic level, in an official institution located in the Jagua de Ibirico carboniferous area of the department of Cesar (Colombia).

A set of didactic strategies focused on mathematical modeling are implemented, with which the students begin with a scheme of problem situations in their context until the construction of a mathematical model, centered on the graphic representation where they describe each situation, comparing each of they and thus they are familiar or morning, to 
solve some real or hypothetical situation that is common in their daily lives.

Likewise, these models may have problems that are interdisciplinary solved and that their relationship is transversal to different areas of knowledge.

With the design and implementation of these strategies for the teaching and learning of statistics, the teacher goes from being a traditional educator of the exact sciences to having a teacher-researcher role and the student assumes a learning rhythm as proposed.

The data obtained provided us with information that corroborates the initial hypothesis about school performance, since they are useful to the process of teaching primary school children.

Theoretical lines have been developed around modeling with the same paradigm and different profiles based on the theories of Bassanezi (2002) and Biembengut \& Hein (2004). Which propose the construction of models based on the context and the social and cultural environment of the student.

The Ministry of National Education $(1998,2006)$ makes a very important contribution of how significant the modeling process is and it involves them in the solution of real problems that can later be mathematized and in general that those learnings are significant. In that sense, Villa (2007), which contemplates the modeling process in accordance with the basic standards of competencies and in our case, strengthens the knowledge tests in addition to the appropriation of basic learning rights.

Likewise, in the subsequent test, a higher performance of each student was found. Therefore, it has been possible to generate spaces for communication with classroom teachers, where they have reflected on the different strategies used in the classroom to achieve better student learning and in turn generates a challenge in each of them in relation to the role Teacher research.

Keywords: mathematical modeling, teaching, pedagogical strategies.

\section{RESUMO}

Este artigo socializa a experiência de pesquisa extraída do projeto "Ensinar estatística: um desafio enfrentado pela modelagem matemática" financiado pela Universidade Popular de Cesar em sua chamada de 2018, aplicada em 2019 com alunos da $5^{a}$ série do ensino fundamental, em um instituição oficial localizada na área carbonífera de Jagua de Ibirico, no departamento de Cesar (Colômbia).

Um conjunto de estratégias didáticas focadas na modelagem matemática é implementado, com o qual os alunos começam com um esquema de situações problemáticas em seu contexto até a construção de um modelo matemático, centrado na representação gráfica em que descrevem cada situação, comparando cada uma delas. eles e, portanto, são familiares ou matinais, para resolver alguma situação real ou hipotética comum em suas vidas diárias.

Da mesma forma, esses modelos podem ter problemas resolvidos interdisciplinarmente e que sua relação é transversal a diferentes áreas do conhecimento.

Com o desenho e a implementação dessas estratégias para o ensino e aprendizado de estatística, o professor deixa de ser um educador tradicional das ciências exatas para ter um papel de professor-pesquisador e o aluno assume um ritmo de aprendizado, conforme proposto.

Os dados obtidos nos forneceram informações que corroboram a hipótese inicial sobre o desempenho escolar, uma vez que são úteis no processo de ensino de crianças do ensino fundamental. 
Linhas teóricas foram desenvolvidas em torno da modelagem com o mesmo paradigma e perfis diferentes, com base nas teorias de Bassanezi (2002) e Biembengut \& Hein (2004). Que propõem a construção de modelos baseados no contexto e no ambiente social e cultural do aluno.

O Ministério da Educação Nacional $(1998,2006)$ dá uma contribuição muito importante de quão significativo é o processo de modelagem e os envolve na solução de problemas reais que posteriormente podem ser matematizados e, em geral, que esses aprendizados são significativos. Nesse sentido, Villa (2007), que contempla o processo de modelagem de acordo com os padrões básicos de competências e, no nosso caso, fortalece os testes de conhecimento, além da apropriação dos direitos básicos de aprendizagem.

Da mesma forma, no teste subsequente, foi encontrado um desempenho superior de cada aluno. Portanto, foi possível gerar espaços de comunicação com os professores em sala de aula, onde eles refletiram sobre as diferentes estratégias utilizadas na sala de aula para alcançar melhor aprendizado dos alunos e, por sua vez, gera um desafio em cada um deles em relação ao papel Pesquisa de professores.

PALAVRAS-CHAVE: modelagem matemática, ensino, estratégias pedagógicas.

\section{INTRODUCCIÓN}

En este capítulo se hace un breve recuento sobre los temas de la modelación matemática como estrategia didáctica y la enseñanza del análisis de gráficas estadísticas, dichas referencias nos permiten comprender los avances y las dificultades que se han venido observando por algunos investigadores sobre estas problemáticas.

La modelación matemática como herramienta didáctica para la enseñanza de las matemáticas garantiza que los educandos logren el aprendizaje a través del trabajo cooperativo, buscando información y que se refuerza mediante el uso de nuevas tecnologías puesto que se forma matemáticamente con situaciones del contexto social, económico y cultural del educando.

Através de un proceso de búsqueda bibliográfica, la temática de la estadística ha sido un punto crítico en investigación y de experimentación además la enseñanza del análisis de gráficos estadísticos, es tratada con poca frecuencia dentro de los trabajos investigativos realizados en el país.

Existen trabajos que abordan la misma temática objeto de esta propuesta. Los trabajos son los siguientes:

Biembengut \& Hein (2004) afirman que la modelación, como método de enseñanza que permite generar un modelo matemático, definido éste como un conjunto de símbolos y relaciones matemáticas que representan un fenómeno real o situación problema y este modelo se puede expresar a través de fórmulas o expresiones numéricas, gráficos 0 formas geométricas, ecuaciones algebraicas, programas computacionales, entre otros para cualquier nivel de escolaridad, en nuestro caso lo aplicamos en el grado 5 de básica primaria.

Villa (2007) afirma que la modelación como estrategia didáctica considera una serie de variables que permiten describir, experimentar y representar una situación en sus diferentes etapas hasta llegar una validación del modelo. Por lo cual no el motivo es dar una mayor significación al proceso de enseñanza.

En ese sentido los aportes desde la modelación hacia los docentes y estudiantes de la I.E Luis Carlos Galán Sarmiento es muy importante, destacamos como a partir de la formulación de un problema sencillo se pueden considerar 
estrategias significativas de conocimiento en cualquier área, con ellas generar aportes en investigación y mayor compromiso por parte de los jóvenes.

Teniendo en cuenta los resultados bajos en las pruebas Saber, la propuesta investigativa se centra en el mejoramiento cognoscitivo de los educandos y en el análisis de gráficos estadísticos contextualizados.

\section{METODOLOGÍA}

Inicialmente se realizó una entrevista donde participación docente y estudiantes para indagar sobre las dificultades presentes en el proceso de enseñanza de las matemáticas en la institución, haciendo hincapié en varios tipos de preguntas como por ejemplo:

De los temas que explica tu profesor de matemáticas entiendes:
A. Nada
B. Sólo algunas cosas
C. Casi todo
D. Todo

En relación a lo anterior, podemos mencionar que parte de los estudiantes tienen ideas confusas y/o erróneas de conceptos básicos en el grado 5. Por su parte en los maestros por no tener la formación profesional en el área de matemáticas presentan dificultades al momento de la preparación de sus clases lo cual repercute en problemas comportamentales de los estudiantes.

De igual manera los docentes reconocen lo complejo en la enseñanza de la estadística en su mayoría de temas y por tal razón priorizamos actividades pertinentes que promovieran el pensamiento aleatorio y sistema de datos con el uso de la modelación matemática en cada uno de los procesos matemáticos del área de estadística.

Implementamos actividades propicias centradas en la modelación, lideradas por Ministerio de educación nacional a través del programa Todos a Aprender; una de ella llamada centro de aprendizaje "La Salamandra" que consta de un material manipulativo donde se desarrollan situaciones hipotéticas que permiten abordar el Derecho básico de aprendizaje (DBA) el cual se evidencia a continuación: formular preguntas que requieren comparar dos grupos de datos, para lo cual recolecta, organiza y usa tablas de frecuencia, gráficos de barras, circulares, de línea, entre otros. Analiza la información presentada y comunica los resultados. (MEN, 2017)

El eje central de dicha actividad es asociar situaciones reales e hipotéticas de los niños hacia la construcción de un modelo como es el diagrama circular, donde los niños pueden conjeturar a partir de los datos recolectados para así planificar, organizar y simbolizarlos en forma gráfica; con esto se logra comparar las diferentes maneras de representar la información recolectada.

A continuación, propone una serie de ejercicios contextualizados que permiten ejercitar los conceptos, proponer alguna otra situación donde intervengan gráficos estadísticos y posterior a ello puedan solucionar situaciones de aplicación donde la transversalidad juegue un papel esencial entre las áreas del saber.

Por lo tanto, se logró evidenciar cambios satisfactorios a nivel conceptual donde los estudiantes podían diferenciar las distintas formas de representación de la información y crear hábitos de estudio en los estudiantes, lo cual repercute en la organización de la información a través de tablas estadísticas además de lograr una mayor atención y concentración en clases. 
De igual manera el estudiante puede analizar la información suministrada e interpretar los resultados, además de hacer conjeturas a partir de las distintas representaciones de un conjunto de datos.

\section{RESULTADOS}

A la par de estas actividades se aplicó un instrumento diagnóstico para observar los desempeños en estadística. Analizando una de las preguntas de la prueba en mención, tomamos como muestra un curso con 36 estudiantes del grado 5 en la sede principal y se obtuvieron los siguientes resultados:

Fig 1: Relación de aciertos vs $\mathrm{N}^{\circ}$ de preguntas de la prueba diagnostica

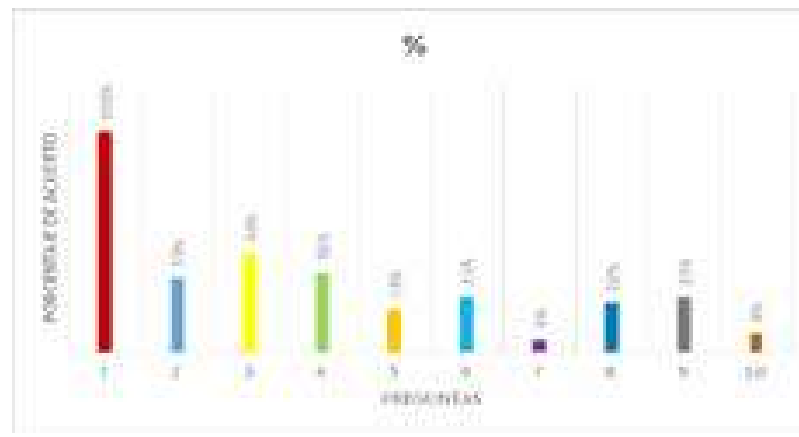

Fuente: Elaboración Propia

A partir del grafico anterior podemos observar el rendimiento de los estudiantes en relación al instrumento diagnóstico, donde se evidencia generalmente un nivel bajo por lo cual las metodologías aplicadas no son las más adecuada así mismo en los indicadores externos de la institución el comportamiento ha sido el mismo.

Podemos mencionar los resultados de la prueba saber en concordancia con el instrumento afirma el aumento de desempeños insuficientes de esta institución educativa. Los cuales se evidencia a continuación:

Fig 2: Niveles de desempeño por año en el área de matemáticas en el periodo 2014-2017

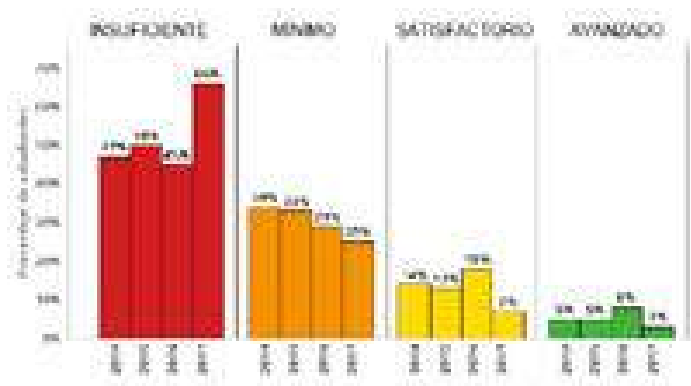

Fuente: Instituto Colombiano para la evaluación de la educación (ICFES.)

El grafico anterior, nos muestra la tendencia de resultados bajos en el periodo (2014-2017) demostrando que las estrategias para la enseñanza repercuten en resultados esperados.

El reto para el maestro de la institución es lograr el porcentaje de desempeños insuficientes y llevar a sus educandos a un nivel satisfactorio y/o avanzado, lo cual no es un reto fácil de cumplir debido a muchos obstáculos presentes en el proceso de enseñanza; sobre todo llamar la atención en la estadística donde la enseñanza se basa en algoritmos y situaciones alejadas de la realidad.

Fig 3: Porcentaje de aciertos posterior a la metodología implementada

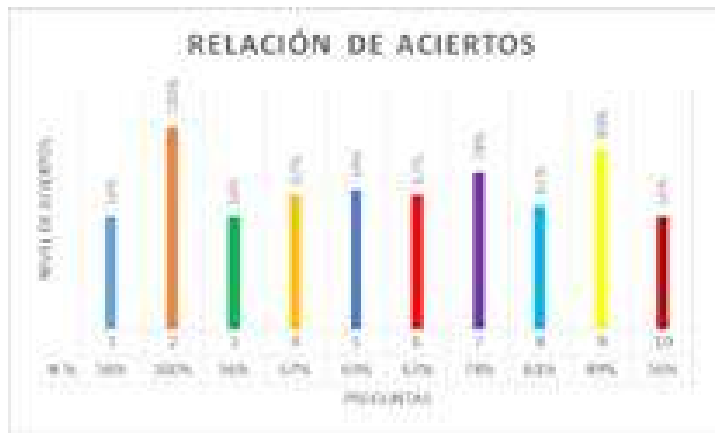

Fuente: Elaboración Propia

Justamente con la metodología aplicada posteriormente encontramos un mayor rendimiento de los estudiantes en general, lo cual ha repercutido en el cambio académico y comportamental de los estudiantes. 


\section{CONCLUSIONES}

Los objetivos propuestos en esta investigación son grandes debido a las dificultades presentes en cada una de las instituciones educativas en el país, con lo cual se ha logrado contribuir de manera significativa considerando los resultados mostrados anteriormente.

A pesar de los esfuerzos de los docentes los resultados en las pruebas externas son muy notorios en los niveles bajo y básico es necesario unificar criterios de evaluación orientados en el diseño, implementación y evaluación de estrategias pertinentes a la enseñanza y aprendizaje de la estadística.

En consecuencia los lineamientos curriculares en matemáticas reconocen 5 procesos matemáticos y la modelación es uno de ellos poco abordado por los docentes en su labor y corrobora que la modelación matemática es una estrategia didáctica para el proceso de enseñanza - aprendizaje de las matemáticas y útil para la estadística

El rol investigativo en el aula garantiza resultados en la práctica pedagógica además que en los test o pruebas aplicadas denoten éxito o fracaso de las metodologías en aula debido a la relación que tienen estas con cada una de las competencias y/o desempeños del estudiante. Por lo cual es relevante hacer seguimiento de las propuestas que sugiere el ministerio de educación nacional

Así mismo podemos recalcar como sugerencia a los maestros y directivos de las instituciones educativas implementar estas estrategias que se encuentran al alcance de todos y son pertinente al abordar conceptos de las ciencias y matemáticas.

Por último, distintos investigadores plantean que la estadística y su enseñanza requieren un manejo adecuado de la información traducida a datos que no son sólo números, sino números propios de un contexto; este logra dar una mayor significación y debe ser tenido en cuenta a la hora de su enseñanza en las distintas actividades dentro del aula.

A manera de conclusión se puede decir que las actividades permitieron motivar a los docentes a realizar nuevas estrategias de enseñanza en estadística, donde los principales actores son los estudiantes pues se encontraron motivados debido a las metodologías implementadas en las clases puesto que participaron activamente en cada una de estas.

\section{REFERENCIAS BIBLIOGRÁFICAS}

Bassanezi, (2002). La enseñanza y el aprendizaje con la modelización matemática: una nueva estrategia

Biembengut \& Hein (2004). Modelación matemática y los desafíos para enseñar matemática.

Instituto Colombiano para la evaluación de la educación (2017). Resultados pruebas saber $3^{\circ}, 5^{\circ}$ y $9^{\circ}$. Recuperado de http://www2. icfesinteractivo.gov.co/ReportesSaber359/

Ministerio de Educación Nacional, (1998). Lineamientos curriculares en matemáticas. Bogotá: Autor.

Ministerio de Educación Nacional, (2006). Estándares básicos de competencias en lenguaje, matemáticas, ciencias y ciudadanas. Bogotá: Autor.

Ministerio de Educación Nacional (2017). Derechos básicos de aprendizaje (DBA) 2da versión. Recuperado de http://aprende. colombiaaprende.edu.co/siemprediae/93226

Villa J. (2007). La modelación como proceso en el aula de matemáticas: un marco de referencia y un ejemplo. Recuperado de https://www. redalyc.org/pdf/3442/344234312004.pdf 